Article

\title{
Optical Characterization of AlAsSb Digital Alloy and Random Alloy on GaSb
}

\author{
Bor-Chau Juang ${ }^{1, *}$, Baolai Liang ${ }^{2}$, Dingkun Ren ${ }^{1}$ (D), David L. Prout ${ }^{3}$, Arion F. Chatziioannou ${ }^{3}$ \\ and Diana L. Huffaker ${ }^{1,2}$ \\ 1 Department of Electrical Engineering, University of California, Los Angeles, CA 90095, USA; \\ dingkun.ren@ucla.edu (D.R.); huffaker@ee.ucla.edu (D.L.H.) \\ 2 California NanoSystems Institute, Los Angeles, CA 90095, USA; bliang@cnsi.ucla.edu \\ 3 Department of Molecular and Medical Pharmacology, Crump Institute for Molecular Imaging, \\ University of California, Los Angeles, CA 90095, USA; DProut@mednet.ucla.edu (D.L.P.); \\ ArChatziioann@mednet.ucla.edu (A.F.C.) \\ * Correspondence: bcjuang@ucla.edu; Tel.: +1-310-825-8592
}

Academic Editor: Paul J. Simmonds

Received: 9 September 2017; Accepted: 15 October 2017; Published: 18 October 2017

\begin{abstract}
III-(As, Sb) alloys are building blocks for various advanced optoelectronic devices, but the growth of their ternary or quaternary materials are commonly limited by spontaneous formation of clusters and phase separations during alloying. Recently, digital alloy growth by molecular beam epitaxy has been widely adopted in preference to conventional random alloy growth because of the extra degree of control offered by the ordered alloying. In this article, we provide a comparative study of the optical characteristics of AlAsSb alloys grown lattice-matched to GaSb using both techniques. The sample grown by digital alloy technique showed stronger photoluminescence intensity, narrower peak linewidth, and larger carrier activation energy than the random alloy technique, indicating an improved optical quality with lower density of non-radiative recombination centers. In addition, a relatively long carrier lifetime was observed from the digital alloy sample, consistent with the results obtained from the photoluminescence study.
\end{abstract}

Keywords: digital alloy; random alloy; epitaxy; molecular beam epitaxy; ternary alloy; aluminum arsenide antimonide; gallium antimonide; photoluminescence

\section{Introduction}

Sb-based compound semiconductors are attractive for advanced optoelectronic applications because of their capability to form ternary and quaternary alloys that allow flexible bandgap engineering, which can be grown lattice-matched to common substrates such as InAs, InP, and GaSb [1-5]. For example, high $\mathrm{Al}$-content alloys with large bandgaps that contain $\mathrm{As}$ and $\mathrm{Sb}$ are often used as window layers or active regions for photodetectors, lasers, and thermophotovoltaics [6-10]. Normally, mixed-anion alloys rely on random alloy (RA) growth in which direct deposition of atoms on the sample surface with carefully tuned growth parameters to achieve the targeted alloy composition. Unfortunately, due to the non-unity incorporation coefficients of volatile group $\mathrm{V}$ elements, the epitaxy of mixed-anion alloys is much more complicated than that of alloys with only one group $\mathrm{V}$ element. The wide miscibility gap of these alloys can easily create undesired crystalline disorders, e.g., As/Sb clusters and phase separation [11,12]. As an alternative, Zhang has proposed modulated molecular beam epitaxy (MMBE) [13], known as the digital alloy (DA), for III-(As, Sb) ordered alloys. The DA growth has been shown to offer a precise control over alloy composition and reproducibility against RA growth $[14,15]$. Although such a technique has been widely implemented to facilitate growths of III-(As, Sb) alloys in device applications [10,16-18], a direct comparison of the material properties of those alloys grown by DA and RA has not been reported. 
In this work, we provide a detailed material analysis of lattice-matched $\mathrm{AlAs}_{0.08} \mathrm{Sb}_{0.92}$ (AlAsSb, hereafter) DA and RA grown on GaSb substrates, and unveil the real strength of DA over RA by comparing heterointerfaces, optical characteristics, and minority carrier lifetimes. We believe this fundamental material research will offer a deeper insight into both growth techniques, and trigger further validating work and device applications.

\section{Experiments}

The AlAsSb samples were deposited on lightly n-doped GaSb (001) substrate using a Veeco Gen930 solid-source MBE reactor. Prior to the material growth, the GaSb substrate temperature was raised to $535{ }^{\circ} \mathrm{C}$ for $20 \mathrm{~min}$ under a $\mathrm{Sb}_{2}$ overpressure to desorb surface oxide. After oxide removal, the substrate was cooled to $510{ }^{\circ} \mathrm{C}$ to grow an unintentionally-doped GaSb buffer. A nominal $600 \mathrm{~nm}$-thick AlAsSb layer was then grown at the same substrate temperature, followed by a $20 \mathrm{~nm}$ $\mathrm{GaSb}$ cap layer to prevent it from rapid oxidation in air. The DA sample was realized by periodically alternating the $\mathrm{As}$ and $\mathrm{Sb}$ shutter while maintaining a steady $\mathrm{Al}$ flux during deposition, resulting in AlSb/AlAs equivalent sequence of 4.0/0.8 MLs. Additional 4.0 MLs of AlSb was used prior to the transition to GaSb cap layer. On the other hand, the RA sample was grown by keeping $\mathrm{Al}$, As, and $\mathrm{Sb}$ shutters open simultaneously, followed by the $\mathrm{GaSb}$ cap layer without growth interruption. The substrate temperature was monitored by an optical pyrometer and calibrated by the change in the surface reconstruction of GaSb from $(1 \times 3)$ to $(2 \times 5)$ using reflection high-energy electron diffraction (RHEED) equipped with the MBE reactor [19]. Separate campaigns of DA and RA samples were grown to obtain their lattice-matching condition, characterized using peak separation from the X-ray diffraction (XRD) scans. The two samples were kept with the identical layer structure and minimal difference in alloy composition to enable a fair comparison. High-resolution triple-axis XRD (HRXRD) measurement (D1, Bede) was performed by inserting an analyzer crystal to define the measurement resolution via a narrow acceptance angle $\left(0.0033^{\circ}\right.$ or $\left.11^{\prime \prime}\right)$. Cross-sectional high-resolution transmission electron microscopy (HRTEM) was performed using FEI Titan TEM. Photoluminescence (PL) characterization was conducted using a $532 \mathrm{~nm}$ solid-state laser to investigate the optical properties of the AlAsSb DA and RA samples. Samples were mounted in the Advanced Research System DE-204 closed-cycle cryostat for temperature-dependent measurement. A silicon photodetector (Model 2151, New Focus, San Jose, CA, USA) was used for steady-state PL measurement via standard lock-in technology. For time-resolved photoluminescence (TRPL), the samples were excited by the NKT Photonics SuperK Extreme laser running at $532 \mathrm{~nm}$ with $20 \mathrm{ps}$ pulse-width. A silicon single-photon avalanche diode from Micro Photon Devices was used to record the TRPL signals. The system time resolution, i.e., instrument response function (IRF), is about $20 \mathrm{ps}$ as extracted from signals of the direct reflection of the pulsed laser.

\section{Results and Discussion}

The HRXRD $\omega-2 \theta$ scans of the AlAsSb DA and RA samples exhibit a single peak of the epitaxial layers, inseparable from the GaSb substrate peak, as shown in Figure 1. The full-widthat-half-maximum (FWHM) values are $18^{\prime \prime}$ and $21^{\prime \prime}$ for the AlAsSb DA and RA samples, respectively, compared to a value of $16^{\prime \prime}$ for the GaSb substrate. This clearly shows that the epitaxial layers are lattice-matched to the substrates, and the compositional fluctuation is negligible. 


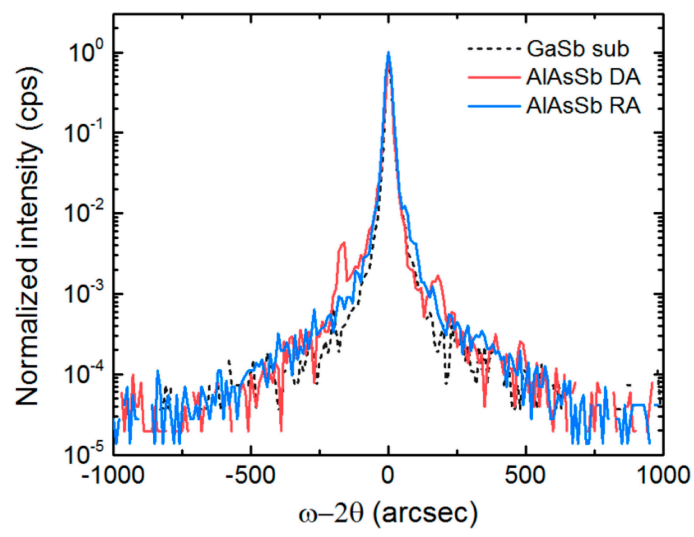

Figure 1. HRXRD $\omega-2 \theta$ scans for the AlAsSb DA and RA samples. The $\omega-2 \theta$ scan of the GaSb substrate is also shown as a reference.

The cross-section TEM of the AlAsSb DA and RA samples is shown in Figure 2. Both samples exhibit ordered phase in AlAsSb based on the presence of the satellite spots in the electron diffraction pattern. The DA sample exhibits no signs of phase separation in the AlAsSb film, as shown in Figure 2a, and the alternating shutter sequence does not produce modulated contrast as found in superlattice growth (Figure 2c). On the other hand, the bulk part of the AlAsSb film in the RA sample is also uniform, whereas a relatively rough $\mathrm{GaSb} / \mathrm{AlAsSb}$ interface is observed (Figure 2c,d). Some bright contrast features parallel to the growth front has been found in the RA sample, likely related to the sample preparation.

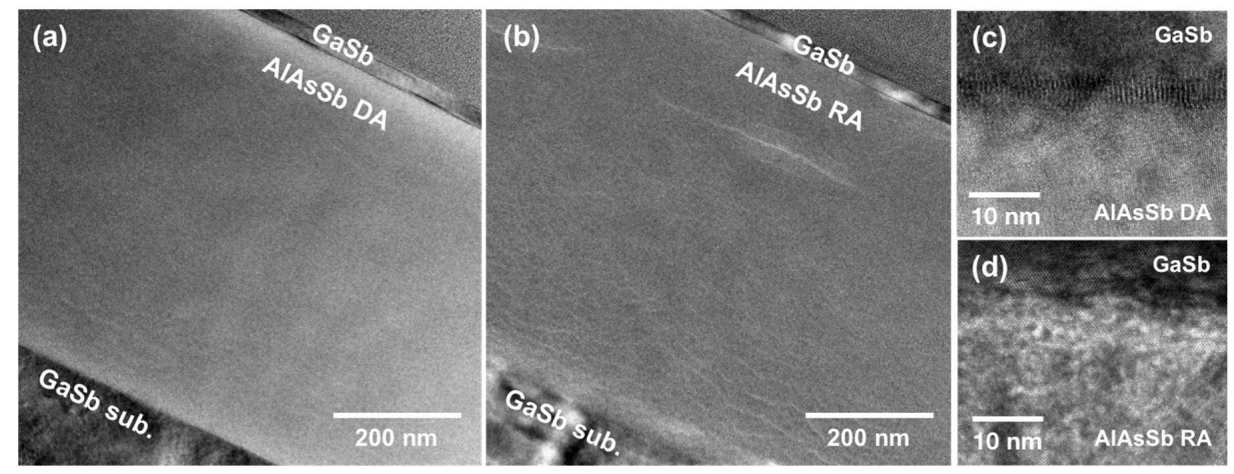

Figure 2. Cross-section TEM images for the AlAsSb (a) DA and (b) RA samples, and (c,d) show the HRTEM images of the GaSb / AlAsSb interfaces.

Low-temperature photoluminescence (PL) spectra of the DA and RA samples are shown in Figure 3a. Both spectra show similar features, including an anomalous emission around $1.29 \mathrm{eV}$ (DL), a narrow peak at $1.6 \mathrm{eV}\left(\mathrm{E}^{\mathrm{X}}\right)$, and a sideband peaking around $1.84 \mathrm{eV}\left(\mathrm{E}^{\mathrm{T}}\right)$, which can be seen more clearly in Figure 3b. In general, the DA sample exhibits stronger luminescence than the RA sample with a considerable difference for the $\mathrm{E}^{\mathrm{X}}$ peak. The PL peaks located at higher energies are most likely due to band-to-band transition. For an indirect bandgap material, the low-temperature optical transition is known to be dominated by the indirect bands, i.e., $X$ band in AlAsSb. Following these rules, we identified the strong emission at $1.6 \mathrm{eV}$ as being the carrier recombination from the $X$ band, and the sideband peaking at $1.84 \mathrm{eV}$ is likely due to the transition from the $\Gamma$ band. In Figure $4 \mathrm{a}$, temperature-dependent $\mathrm{E}^{\mathrm{X}}$ peak energies are fitted with a hyperbolic cotangent relation [20],

$$
E_{g}(T)=E_{g}(0)-S\langle\hbar \omega\rangle\left(\operatorname{coth}\left(\frac{\langle\hbar \omega\rangle}{2 k_{B} T}\right)-1\right)
$$


where $E_{g}(0)$ is the bandgap energy at $0 \mathrm{~K}, S$ is the dimensionless coupling coefficient, $\langle\hbar \omega\rangle$ is the average phonon energy, and $k_{B}$ is the Boltzmann constant. The fitting parameters are shown in Table 1. The fittings show good agreement with the experimental data, and the extracted values of $\langle\hbar \omega\rangle$ also match the calculated optical phonon energies [21]. The DA sample also exhibits a smaller bandgap energy than the RA sample, likely due to the ordering effect [22]. Similar results have been observed in other quaternary materials, the DA growths of which exhibit a relatively small bandgap energy, within $2-3 \%$ of that of the RA growth $[15,23]$. On the other hand, DL peaks display an anomalous temperature dependence with respect to the $\mathrm{E}^{\mathrm{X}}$ peak, which will be discussed later in the text.
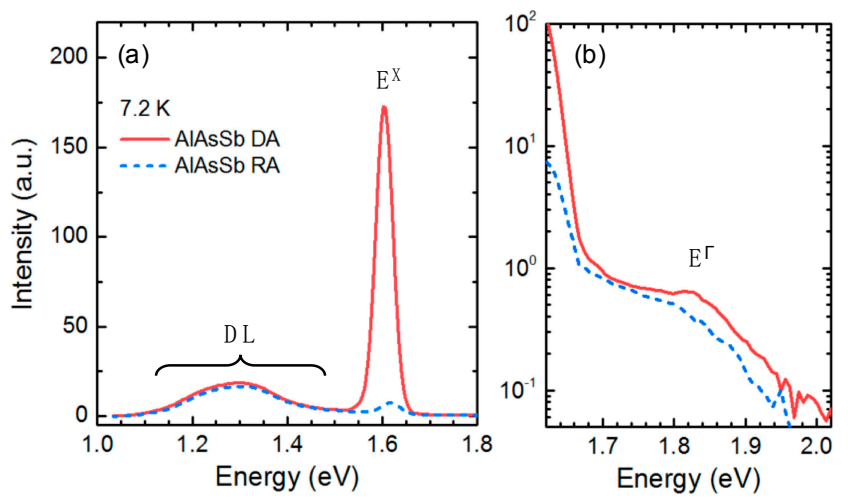

Figure 3. (a) Low-temperature PL spectra of the AlAsSb DA and RA samples at $7.2 \mathrm{~K}$; and (b) the $\Gamma$ band transition with a logarithmic scale.
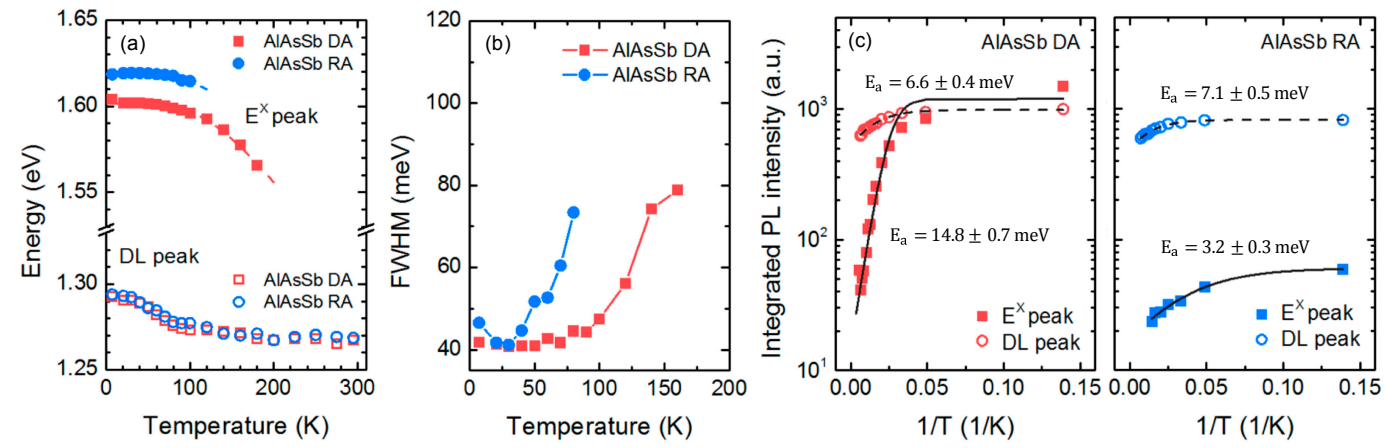

Figure 4. Temperature-dependent measurement of (a) peak energies; (b) peak FWHM; and (c) integrated PL intensities of the $\mathrm{E}^{\mathrm{X}}$ and DL peaks for the AlAsSb DA and RA samples.

Table 1. Parameters extracted by fitting experimental data of the AlAsSb DA and RA samples.

\begin{tabular}{cccc}
\hline Sample & $\boldsymbol{E}_{\boldsymbol{g}}(\mathbf{0})$ & $S$ & $\langle\hbar !\rangle$ \\
\hline AlAsSb DA & $1.602 \mathrm{eV}$ & 4.89 & $38.1 \pm 3.4 \mathrm{meV}$ \\
AlAsSb RA & $1.619 \mathrm{eV}$ & 3.00 & $32.3 \pm 9.2 \mathrm{meV}$ \\
\hline
\end{tabular}

Temperature-dependent FWHM of the $\mathrm{E}^{\mathrm{X}}$ peaks for both samples are shown in Figure $4 \mathrm{~b}$, and these are anticipated to be relatively high, due to the involvement of multiple phonons in the indirect band transition. However, the DA sample does not exhibit broader PL emissions, such as those found in as-grown AlGaInP systems [23], suggesting that there is no sign of defects originating from the large numbers of interfaces. The FWHM is approximately the same (40-45 meV) at low temperatures $(<40 \mathrm{~K})$, whereas the DA sample shows consistently smaller FWHM than the RA sample at elevated temperatures, suggesting a better crystalline quality.

Integrated PL intensities of the $\mathrm{E}^{\mathrm{X}}$ and DL peaks were studied, and the results are shown in Figure $4 \mathrm{c}$. It can be seen that $\mathrm{E}^{\mathrm{X}}$ peak intensities drop quickly below $40 \mathrm{~K}$ with increasing temperature. 
In spite of the similar temperature-dependence of DL peaks between the DA and RA samples, a marked difference in the $\mathrm{E}^{\mathrm{X}}$ peak can be observed. The activation energies of the $\mathrm{E}^{\mathrm{X}}$ peak can be extracted using the Arrhenius equation [24],

$$
I(T)=\frac{I_{0}}{1+C_{0} \exp \left(-E_{a} / k_{B} T\right)}
$$

where $I_{0}$ is the integrated PL intensity at $0 \mathrm{~K}, C_{0}$ is the rate constant, and $E_{a}$ is the activation energy. The $E^{X}$ peak shows a much higher activation energy for the DA sample than for the RA sample, suggesting that the carrier-quenching process is much faster in the RA sample. The loss of carriers is likely due to phonon scattering, assisted by crystalline disorders such as phase separation during the RA growth. In addition, the non-uniformity of alloy composition could also enhance non-radiative recombination. On the other hand, the similar activation energies of the DL peaks indicate a common mechanism, regardless of the growth methods.

The origins of photon emission have been characterized using the power law, $I \propto P^{k}$., where $I$ is the integrated PL intensity and the exponent $k$ is a characteristic coefficient. The fitted $k$ value depends on the radiative recombination mechanisms, where an exciton transition usually gives $1<k<2$ and transitions due to free-to-bound or donor-acceptor pair recombination give $k<1$ [25-27]. In Figure 5a, fitting the data to $\mathrm{E}^{\mathrm{X}}$ peaks exhibits $k=1.22$ and 1.3 for the DA and RA samples, respectively, confirming that the transition is dominated by band-to-band recombination, as discussed earlier. On the other hand, fitting to the DL peak for both samples yields $k=0.66$ and 0.71 for the DL and RA samples, respectively, suggesting that the radiative recombination involved states located within the bandgap. The appearance of the low-energy DL peak is common to both samples at all temperatures studied, regardless of the growth methods, and shows a relatively weak temperature dependence for the peak energies and integrated PL intensities, as shown in Figure 4a,c. As a result, the DL peak could presumably be associated with unresolved transitions from deep level states in AlAsSb [28].

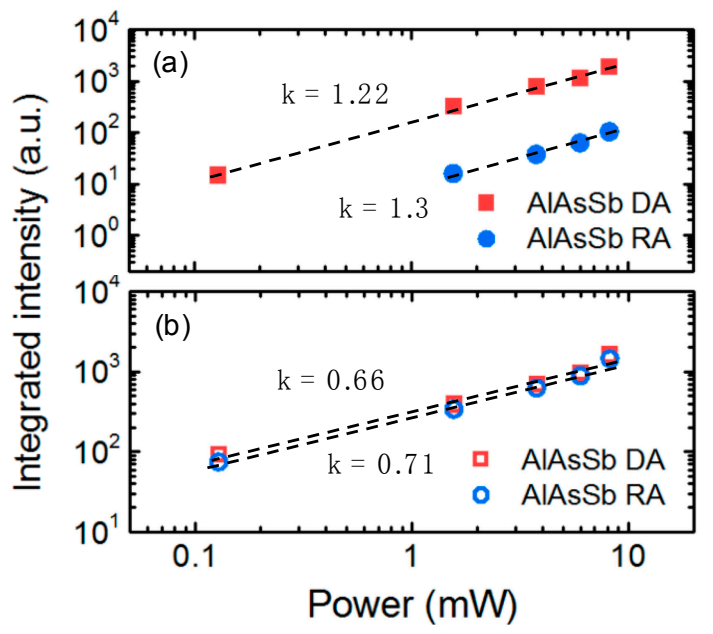

Figure 5. PL peak energies of (a) $\mathrm{E}^{\mathrm{X}}$ peak and (b) DL peak for both DA and RA samples as a function of laser excitation power.

TRPL decay from the $\mathrm{E}^{\mathrm{X}}$ peak transition of the DA and RA samples are measured and pumped under the same excitation power, as shown in Figure 6. The decay tails of both samples are best fitted using a single exponential relation [29],

$$
I(T)=A_{0}+A_{1} \exp (-t / \tau)
$$


where $I(T)$ is the counted PL intensity, $A_{0}$ and $A_{1}$ are fitting parameters, and $\tau$ is the carrier lifetime. The IRF is also shown as an indication of the system time resolution. The DA sample shows a longer minority carrier lifetime in comparison to the RA sample, providing further evidence that the DA sample has fewer non-radiative recombination centers.

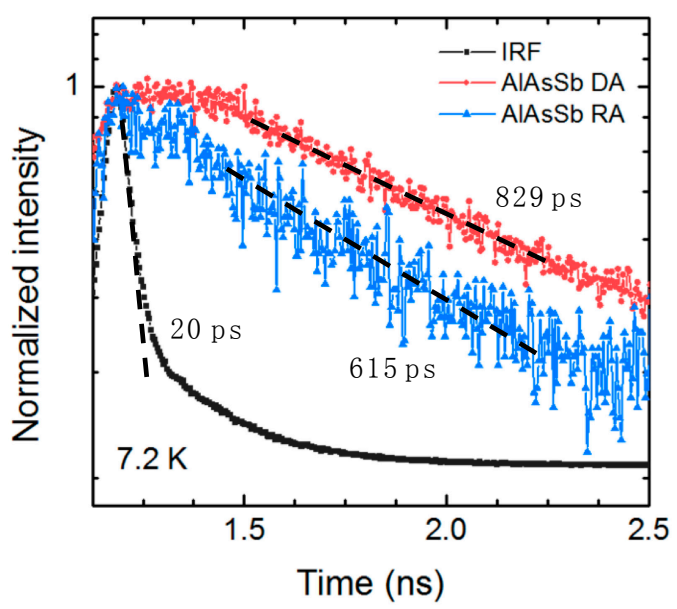

Figure 6. The TRPL data for the DA and RA samples of $X$ band transition at 7.2 K. The IRF is also shown in the plot.

\section{Conclusions}

This study investigated the optical characteristics of the AlAsSb lattice-matched to GaSb using DA and RA growth techniques by MBE. Both samples are lattice-matched to GaSb substrates, as verified using HRXRD. The AlAsSb indirect band transition in the DA sample shows consistently stronger PL intensities with smaller FWHM values at elevated temperatures, suggesting that the material is relatively free from non-radiative recombination and phonon scattering induced by phase separation. This is supported by the higher activation energy of carrier quenching, as well as the longer lifetime of the DA sample in comparison to the values for the RA sample. These results provide direct evidence that, compared to conventional RA growth, DA growth could offer a more compositionally uniform III-(As, Sb) alloy with enhanced optical quality, better suited for applications in optoelectronic devices.

Acknowledgments: The authors acknowledge the financial support by the Defense Threat Reduction Agency (DTRA) through award number HDTRA1-14-1-0035 and the support by the CNSI/HP seeding funding (Grant \# 449041-HD-79740). The authors would also like to acknowledge the financial support provided by Sêr Cymru National Research Network in Advanced Engineering and Materials.

Author Contributions: Bor-Chau Juang, Baolai Liang, and Diana L. Huffaker conceived and designed the experiments; David L. Prout and Arion F. Chatziioannou provide scientific discussion; Bor-Chau Juang performed the MBE growth of samples and the optical experiments; Dingkun Ren performed the transmission electron microscopy; Bor-Chau Juang and Baolai Liang analyzed the data; Bor-Chau Juang wrote the original draft.

Conflicts of Interest: The authors declare no conflicts of interest.

\section{References}

1. Dutta, P.S.; Bhat, H.L.; Kumar, V. The physics and technology of gallium antimonide: An emerging optoelectronic material. J. Appl. Phys. 1997, 81, 5821-5870. [CrossRef]

2. Kroemer, H. The $6.1 \AA$ family (InAs, GaSb, AlSb) and its heterostructures: A selective review. Phys. E Low-Dimens. Syst. Nanostruct. 2004, 20, 196-203. [CrossRef]

3. Soibel, A.; Hill, C.J.; Keo, S.A.; Hoglund, L.; Rosenberg, R.; Kowalczyk, R.; Khoshakhlagh, A.; Fisher, A.; Ting, D.Z.Y.; Gunapala, S.D. Room temperature performance of mid-wavelength infrared InAsSb nBn detectors. Appl. Phys. Lett. 2014, 105, 23512. [CrossRef] 
4. Krier, A. Mid-Infrared Semiconductor Optoelectronics; Springer Series in Optical Sciences; Springer: London, UK, 2012; ISBN 9781849965613.

5. Zhao, Z.; Laghumavarapu, R.B.; Simmonds, P.J.; Ji, H.; Liang, B.; Huffaker, D.L. Photoluminescence study of the effect of strain compensation on InAs/AlAsSb quantum dots. J. Cryst. Growth 2015, 425, 312-315. [CrossRef]

6. Xie, J.; Ng, J.S.; Tan, C.H. An InGaAs / AlAsSb Avalanche Photodiode With a Small Temperature Coefficient of Breakdown. IEEE Photonics J. 2013, 5, 6800706. [CrossRef]

7. Wilk, A.; Genty, F.; Fraisse, B.; Boissier, G.; Grech, P.; El Gazouli, M.; Christol, P.; Oswald, J.; Simecek, T.; Hulicius, E.; et al. MBE growth of InAs/InAsSb/AlAsSb structures for mid-infrared lasers. J. Cryst. Growth 2001, 223, 341-348. [CrossRef]

8. Revin, D.G.; Wilson, L.R.; Zibik, E.A.; Green, R.P.; Cockburn, J.W.; Steer, M.J.; Airey, R.J.; Hopkinson, M. InGaAs/AlAsSb quantum cascade lasers. Appl. Phys. Lett. 2004, 85, 3992-3994. [CrossRef]

9. Xie, S.; Tan, C.H. AlAsSb Avalanche Photodiodes With a Sub-mV/K Temperature Coefficient of Breakdown Voltage. IEEE J. Quantum Electron. 2011, 47, 1391-1395. [CrossRef]

10. Woodson, M.E.; Ren, M.; Maddox, S.J.; Chen, Y.; Bank, S.R.; Campbell, J.C. Low-noise AlInAsSb avalanche photodiode. Appl. Phys. Lett. 2016, 108, 81102. [CrossRef]

11. Stringfellow, G.B. Miscibility gaps in quaternary III/V alloys. J. Cryst. Growth 1982, 58, 194-202. [CrossRef]

12. Wang, C.A. Progress and continuing challenges in GaSb-based III-V alloys and heterostructures grown by organometallic vapor-phase epitaxy. J. Cryst. Growth 2004, 272, 664-681. [CrossRef]

13. Zhang, Y.H. Accurate control of As and $\mathrm{Sb}$ incorporation ratio during solid-source molecular-beam epitaxy. J. Cryst. Growth 1995, 150, 838-843. [CrossRef]

14. Maddox, S.J.; March, S.D.; Bank, S.R. Broadly Tunable AlInAsSb Digital Alloys Grown on GaSb. Cryst. Growth Des. 2016, 16, 3582-3586. [CrossRef]

15. Kaspi, R.; Donati, G.P. Digital alloy growth in mixed As/Sb heterostructures. J. Cryst. Growth 2003, 251, 515-520. [CrossRef]

16. Kaspi, R.; Ongstad, A.; Dente, G.C.; Chavez, J.; Tilton, M.L.; Gianardi, D. High power and high brightness from an optically pumped InAs/InGaSb type-II midinfrared laser with low confinement. Appl. Phys. Lett. 2002, 81, 406-408. [CrossRef]

17. Mourad, C.; Gianardi, D.; Malloy, K.J.; Kaspi, R. $2 \mu \mathrm{m}$ GaInAsSb/AlGaAsSb midinfrared laser grown digitally on GaSb by modulated-molecular beam epitaxy. J. Appl. Phys. 2000, 88, 5543-5546. [CrossRef]

18. Bennett, B.R.; Ancona, M.G.; Boos, J.B.; Canedy, C.B.; Khan, S.A. Strained GaSb/AlAsSb quantum wells for p-channel field-effect transistors. J. Cryst. Growth 2008, 311, 47-53. [CrossRef]

19. Bracker, A.S.; Yang, M.J.; Bennett, B.R.; Culbertson, J.C.; Moore, W.J. Surface reconstruction phase diagrams for InAs, AlSb, and GaSb. J. Cryst. Growth 2000, 220, 384-392. [CrossRef]

20. O'Donnell, K.P.; Chen, X. Temperature dependence of semiconductor band gaps. Appl. Phys. Lett. 1991, 58, 2924-2926. [CrossRef]

21. Sela, I.; Bolognesi, C.R.; Kroemer, H. Single-mode behavior of $\mathrm{AlSb}_{(1-x)} \mathrm{As}_{x}$ alloys. Phys. Rev. B 1992, 46, 16142-16143. [CrossRef]

22. Kawamura, Y.; Gomyo, A.; Suzuki, T.; Higashino, T.; Inoue, N. Band-Gap Change in Ordered/Disordered $\mathrm{GaAs}_{1-y} \mathrm{Sb}_{y}$ Layers Grown on (001) and (111)B InP Substrates. Jpn. J. Appl. Phys. 2002, 41, L447. [CrossRef]

23. Kwon, O.; Lin, Y.; Boeckl, J.; Ringel, S.A. Growth and properties of digitally-alloyed AlGaInP by solid source molecular beam epitaxy. J. Electron. Mater. 2005, 34, 1301-1306. [CrossRef]

24. As, D.J.; Schmilgus, F.; Wang, C.; Schöttker, B.; Schikora, D.; Lischka, K. The near band edge photoluminescence of cubic GaN epilayers. Appl. Phys. Lett. 1997, 70, 1311-1313. [CrossRef]

25. Schmidt, T.; Lischka, K.; Zulehner, W. Excitation-power dependence of the near-band-edge photoluminescence of semiconductors. Phys. Rev. B 1992, 45, 8989-8994. [CrossRef]

26. Lieten, R.R.; Bustillo, K.; Smets, T.; Simoen, E.; Ager, J.W.; Haller, E.E.; Locquet, J.P. Photoluminescence of bulk germanium. Phys. Rev. B 2012, 86, 35204. [CrossRef]

27. Wang, G.; Liang, B.; Juang, B.C.; Das, A.; Debnath, M.C.; Huffaker, D.L.; Mazur, Y.I.; Ware, M.E.; Salamo, G.J. Comparative study of photoluminescence from In0.3Ga0.7As/GaAs surface and buried quantum dots. Nanotechnology 2016, 27, 465701. [CrossRef] [PubMed] 
28. Ma, Y.J.; Zhang, Y.G.; Gu, Y.; Xi, S.P.; Chen, X.Y.; Liang, B.; Juang, B.C.; Huffaker, D.L.; Du, B.; Shao, X.M.; et al. Behaviors of beryllium compensation doping in InGaAsP grown by gas source molecular beam epitaxy. AIP Adv. 2017, 7. [CrossRef]

29. Baranowski, M.; Kudrawiec, R.; Syperek, M.; Misiewicz, J.; Sarmiento, T.; Harris, J.S. Time-resolved photoluminescence studies of annealed 1.3- $\mu \mathrm{m}$ GaInNAsSb quantum wells. Nanoscale Res. Lett. 2014, 9, 81. [CrossRef] [PubMed] 